\title{
The accuracy of 3T magnetic resonance cholangiopancreatography in suspected choledocholithiasis
}

\author{
Önder Yeniçeri ${ }^{A, B, D, E}$, Neşat Çullu ${ }^{C, D, E}$, Burak Özşeker ${ }^{A, B, D}$, Emine Neşe Yeniçeri ${ }^{D, E, F}$ \\ Faculty of Medicine, Mugla Sitki Kocman University, Turkey
}

\section{Abstract}

\begin{abstract}
Purpose: The purpose of this study was to investigate interobserver agreement during magnetic resonance cholangiopancreatography (MRCP) evaluation and the sensitivity and specificity of MRCP obtained with 3T scanners in cases of bile duct obstruction.
\end{abstract}

\begin{abstract}
Material and methods: A total of 37 patients who had MRCP and endoscopic retrograde cholangiography (ERCP) were included. Choledochal pathology was divided into two groups regarding the presence of stones as "there is stone or not". MRCPs were performed with a 3-Tesla system using respiratory triggered HASTE technique in axial and coronal planes and with T2 SPACE sequence in the coronal plane. Sensitivity, specificity, negative predictive value (NPV), and positive predictive value (PPV) were calculated separately for each observer. The average of both observers was calculated for comparison with other studies.
\end{abstract}

Results: Thirty-seven patients were in the study population. Agreement between the observers was analysed, and Cohen's $\kappa$ value was evaluated as 0.84 . For two observers, the sensitivity of MRCP was $93 \%$, whereas the specificity was $75 \%$ for the first observer and $62 \%$ for the second.

Conclusions: In this study we found a high level of interobserver agreement in evaluating MRCP. MRCP has a high sensitivity in detecting choledocholithiasis.

Key words: cholelithiasis, ERCP, bile duct, MRCP.

\section{Introduction}

Biliary obstruction may be due to choledocholithiasis, tumours, trauma, or post-surgical strictures. An imaging method is used in conjunction with biochemical parameters for diagnosis. Endoscopic retrograde cholangiography (ERCP) is currently the gold standard diagnostic tool for the imaging of bile ducts [1]. However, the need for general anaesthesia for ERCP creates a disadvantage because it is an invasive and expensive procedure. Magnetic resonance cholangiopancreatography (MRCP) and endoscopic ultrasonography (EUS) are alternatives to ERCP as a method for noninvasive diagnostic imaging of bile ducts. There are several reports indicating that the sensitivity and specificity of MRCP in choledocholithia- sis are $27-90 \%$ and $40-100 \%$, respectively [1-6]. The lack of a concomitant therapeutic approach is a disadvantage of this method; for this reason, MRCP is usually followed by an invasive method. Because some reports show that MRCP does not change the management of suspected choledocholithiasis, the benefit for this group of patients is being questioned $[2,3]$.

In literature, the majority of studies conducted on the sensitivity and specificity of MRCP were performed with $1.5 \mathrm{~T}$ systems due to the widespread use of devices. Initially, SAR problems, coils mismatches, susceptibility artefacts, and penetration failure limited the use of $3 \mathrm{~T}$ scanners [7]. However, recently the use of 3T scanners has increased by overcoming of these problems and with the development of new sequence structures.

\section{Correspondence address:}

Dr. Neşat Çullu, Faculty of Medicine, Mugla Sitki Kocman University, 48000, Mugla, Turkey, phone: +905072030472, e-mail: nesatcullu77@gmail.com

Authors' contribution:

A Study design · B Data collection · C Statistical analysis · D Data interpretation · E Manuscript preparation · F Literature search · G Funds collection 
Table 1. Parameters of sequences used in magnetic resonance cholangiopancreatography

\begin{tabular}{|l|c|c|c|c|} 
& $\begin{array}{c}\text { TR } \\
(\mathrm{msn})\end{array}$ & $\begin{array}{c}\text { TE } \\
(\mathrm{msn})\end{array}$ & $\begin{array}{c}\text { Slice thickness } \\
(\mathrm{mm})\end{array}$ & $\begin{array}{c}\text { FOV } \\
(\mathrm{cm})\end{array}$ \\
\hline Axial HASTE & 1600 & 95 & 5 & $37 \times 29$ \\
\hline Coronal HASTE & 1400 & 108 & 5 & $37 \times 34$ \\
\hline T2 SPACE & 4489 & 702 & 1 & $37 \times 37$ \\
\hline
\end{tabular}

The purpose of this study is to investigate interobserver agreement during MRCP evaluation and the sensitivity and specificity of MRCP obtained with 3T scanners in cases of bile duct obstruction.

\section{Material and methods}

Ethical approval was obtained from our university. Hospital records of 48 patients whose MRCPs were performed in the Radiology Clinic and ERCPs were performed in the Gastroenterology Clinic consecutively with suspected choledocholithiasis were evaluated between May 2017 and October 2017. Seven patients considered with tumour by MRCP and ERCP and diagnosed histopathologically were excluded. Four of the patients with failed ERCP for different reasons were also excluded. In total, 37 patients who had MRCP and ERCP were included. The patients were evaluated by two experienced radiologists. The radiologists were blinded to each other and the diagnosis of the patients.

Choledochal pathology was divided into two groups depending on the presence of stones as "there is stone or not". Extrahepatic bile duct diameters were measured. Gall bladder was examined for the presence of stones. Biochemical parameters such as alanine aminotransferase (ALT), aspartate aminotransferase (AST), gamma-glutamyl transferase (GGT), alkaline phosphatase (ALP), and total bilirubin values were recorded.
MRCP was performed with a 3-Tesla (Siemens Skyra, Erlangen, Germany) system using respiratory-triggered HASTE technique in axial and coronal planes and with a T2 SPACE sequence in the coronal plane. Images of the T2 SPACE sequence were then reconstructed to maximum intensity projection (MIP) images in the coronal plane with 10 degrees (totally 36 images). Sequence parameters are given in Table 1.

ERCP techniques were performed with a side-viewed Pentax ED 3490 duodenoscope (Tokyo, Japan). Bile ducts were cannulated with a guidewire by sphincterotomy. Images were obtained with nonionic contrast materials. In cases of the presence of a stone, it was retrieved with a balloon catheter.

Mann-Whitney $U$ test was used to compare demographic and laboratory data. Cohen's $\kappa$ value was calculated for interobserver agreement. Sensitivity, specificity, negative predictive value (NPV), and positive predictive value (PPV) were calculated separately for each observer. The average of both observers was calculated for comparison with other studies. All statistical analyses were done using SPSS software (IBM SPSS Statistics 20).

\section{Results}

Seven patients who had MRCP-ERCP comparison were diagnosed with tumours and were excluded from the study. Four of the patients who had ERCP failure for other reasons were also excluded. The ERCP failure rate was $8.33 \%$. The reasons for failure were as follows: fibrotic papilla, papillary oedema, the papilla could not be found in one patient with gastroenterostomy, and papilla was placed at the rim of duodenal diverticula in another patient.

Thirty-seven patients were included in the study population. The average age of the study population was 63.51 (14-91) years. Fifteen of the patients were male and 22 were female. The average age of the male patients was 68.67 (20-91) years and of the female patients was 60 (14-91)

Table 2. Demographic, biochemical, and ultrasonographic data of the cases

\begin{tabular}{|l|c|c|c|} 
& $\begin{array}{c}\text { No choledocholithiasis }(n=7) \\
\text { (Median, min, max) }\end{array}$ & $\begin{array}{c}\text { Choledocholithiasis }(n=30) \\
\text { (Median, min, max) }\end{array}$ & $p$ value \\
\hline Age (year) & $56(14-72)$ & $69.50(27-91)$ & 0.029 \\
\hline Total bilirubin $(\mathrm{mg} / \mathrm{dl})$ & $1.70(0.28-7.05)$ & $2.75(0.49-11.45)$ & 0.406 \\
\hline AST $(\mathrm{U} / \mathrm{l})$ & $49(28-257)$ & $65(10-552)$ & 0.815 \\
\hline ALT $(\mathrm{U} / \mathrm{l})$ & $62(20-307)$ & $95(10-587)$ & 0.815 \\
\hline GGT $(\mathrm{U} / \mathrm{l})$ & $225(19-546)$ & $250(20-1397)$ & 0.410 \\
\hline ALP $(\mathrm{U} / \mathrm{l})$ & $102(55-308)$ & $155(23-667)$ & 0.178 \\
\hline Cholelithiasis $(n)$ & 5 & 18 & 12 \\
\hline Cholecystectomies $(n)$ & 2 & $11.65(6-23)$ & $<0.001$ \\
\hline CBD diameter $(\mathrm{mm})$ & $5(3-8)$ & & \\
\hline
\end{tabular}

CBD - common bile duct, min - minimum, max - maximum 
years. Demographic features and laboratory data are summarised in Table 2.

The average time between MRCP and ERCP was 5.46 (1-15) days. The median bile duct size of the seven patients whose MRCP and ERCP results were evaluated as normal was $5 \mathrm{~mm}(3-8 \mathrm{~mm})$. The median bile duct size was $11.65 \mathrm{~mm}(6-23)$ in 30 patients with choledocholithiasis.

The agreement between the observers was analysed, and Cohen's $\kappa$ value was evaluated as 0.84 . For two observers, the sensitivity of MRCP was $93 \%$ whereas the specificity was $75 \%$ for the first observer and $62 \%$ for the second. Sensitivity, specificity, accuracy, PPV, and NPV values are shown in Table 3.

\section{Discussion}

In this study, MRCP was compared with ERCP, and the sensitivity of MRCP in patients with choledocholithiasis was found to be $93 \%$ and specificity $69 \%$. The sensitivity of MRCP is reported as being between $27 \%$ and $100 \%$ in the literature $[2,5,6]$. In our study, the sensitivity of MRCP that
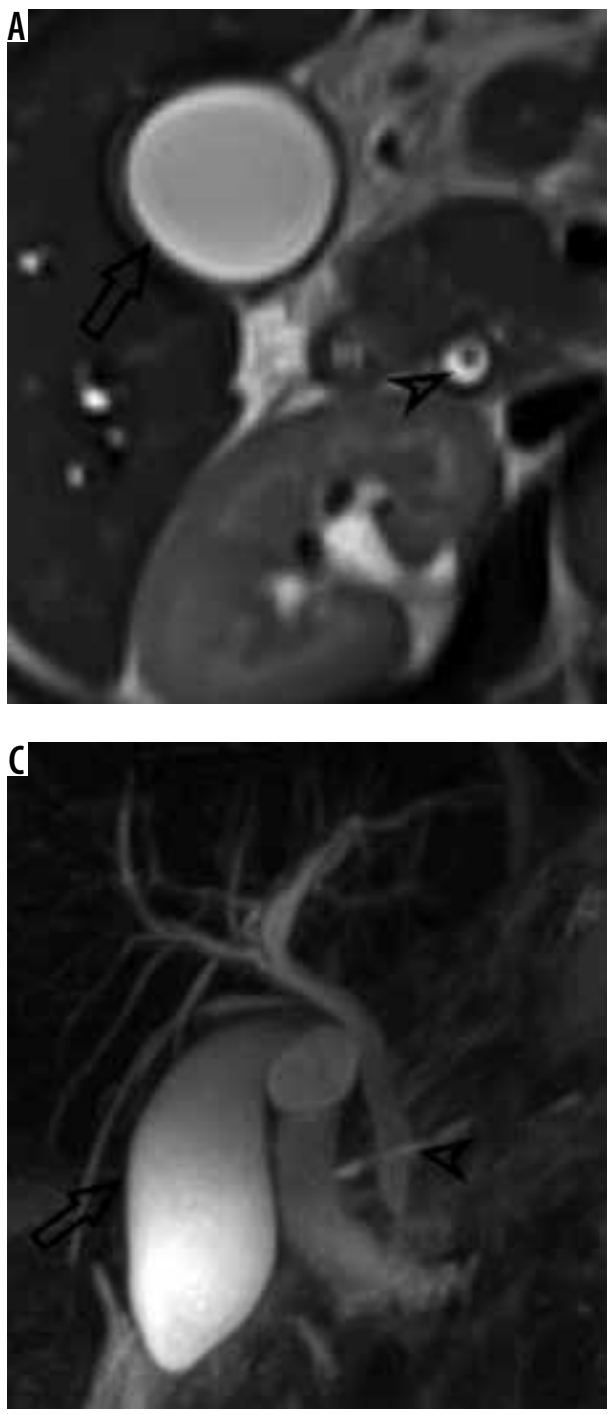

Table 3. Sensitivity, specificity, positive predictive value (PPV), negative predictive value (NPV), and accuracy values both observers

\begin{tabular}{|l|c|c|c|c|c|} 
& $\begin{array}{c}\text { Sensitivity } \\
(\%)\end{array}$ & $\begin{array}{c}\text { Specificity } \\
(\%)\end{array}$ & $\begin{array}{c}\text { PPV } \\
(\%)\end{array}$ & $\begin{array}{c}\text { NPV } \\
(\%)\end{array}$ & $\begin{array}{c}\text { Accuracy } \\
(\%)\end{array}$ \\
\hline Observer 1 & 93 & 75 & 93 & 75 & 89 \\
\hline Observer 2 & 93 & 62 & 90 & 71 & 86 \\
\hline
\end{tabular}

we found was similar to that of the high-level sensitivity groups in studies in the literature. Some studies found the specificity of MRCP to be between $40 \%$ and $100 \%$. In this perspective, in our study, the sensitivity result seems to be in the medium sensitivity group [1,4]. In a comparison of the specificity value with other series, we found a lower value because the first observer reported suspected stone in the bile ducts of two patients and the second observer in three patients. Both observers interpreted false positive stones in the same cases. The time between MRCP and ERCP of these patients was 3-5 days. There was a dark focus in the axial HASTE series in the central distal portion of the bile duct in two patients who had false positive

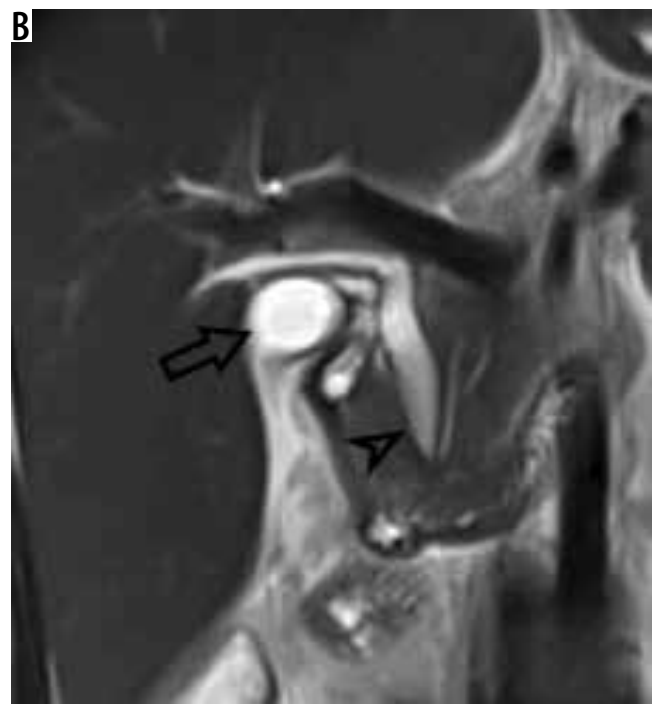

Figure 1. A) There was a hypointensity in axial HASTE series in central portion of distal bile duct (choledoc: arrowhead) (gall bladder: open arrow). $B, C)$ This hypointensity was not seen in coronal HASTE and T2 SPACE MIP images 
Table 4. Sensitivity, specificity, positive predictive value (PPV), and negative predictive value (NPV) reported in the literature for MRCP in patients with suspected choledocholithiasis

\begin{tabular}{|l|c|c|c|c|c|c|c|}
\hline Author & Year & Scanner (Tesla) & $n$ & Sensitivity (\%) & Specificity (\%) & PPV (\%) & NPV (\%) \\
\hline Present study & 2017 & 3T & 37 & 93 & 69 & 91.5 & 73 \\
\hline Makmun [1] & 2017 & 1.5 & 31 & 81 & 40 & 74 & 50 \\
\hline Badger [2]** & 2016 & 1.5 or 3 & 47 & 90 & 86 & 97 & 60 \\
\hline Aydelotte [3] & 2015 & 1.5 & 36 & 90 & 88 & 97 & 64 \\
\hline Polistina [4] & 2015 & 1.5 & 111 & 77.4 & 100 & 100 & 85 \\
\hline Richard* [5] & 2013 & NR & 70 & 27 & 83 & 36 & 77 \\
\hline Demartine [6] & 2000 & 1.5 & 40 & 100 & 95.6 & 92.6 & 100 \\
\hline
\end{tabular}

*In this study, MRCP was compared with intraoperative cholangiography

**In this study, MRCP was compared with intraoperative cholangiography or ERCP

NR - not reported

choledocholithiasis as reported by both of the observers (Figure 1A). This dark focus was not seen in coronal HASTE and T2 SPACE images (Figure 1B-C). It is thought that it could be due to the flow because the location of this false image is in the central distal portion of bile duct and only seen in the axial plane. Millimetric stones and sludge were reported in the ERCP of false negative cases (Table 4).

We also examined the agreement between observers. Cohen's $\kappa$ value was calculated as 0.84 . This value shows that the agreement between the observers is quite good. Although there are studies concerning interobserver agreement of MRCP sequences, there is no adequate evidence and discussion about interobserver agreement of MRCP in patients with suspected choledocholithiasis $[8,9]$.

In the first evaluation of patients with suspected choledocholithiasis, blood tests (ALT, AST, GGT, ALP, and total bilirubin) and transabdominal ultrasonography (TAUS) should be performed [10]. Levels of cholestatic biochemical markers (GGT, ALP, and bilirubin) usually increase gradually, consistent with the duration and severity of biliary obstruction. However, these biochemical tests are not specific to choledocholithiasis and can increase in some other conditions like pancreatitis in the first line. No statistical significance was found in levels of biochemical tests in patients with choledocholithiasis and in patients without choledocholithiasis in this study. In the group of patients without choledocholithiasis, one patient had hepatitis and sludge in the gall bladder, one patient had cholecystitis, and two other patients had pancreatitis. The other three patients were investigated because of complaints that developed after cholecystectomy and the establishment of large bile duct in ultrasonography. The last patient had increased levels in biochemical tests but normalised in the following tests.

Normally the bile duct diameter is smaller than $6 \mathrm{~mm}$ [11]. A mild dilation is reported to be normal due to aging [12]. Biliary obstruction should be suggested when the bile duct diameter is over $8 \mathrm{~mm}$ in patients with normal gallbladder [13]. In the group of patients without choledocholithiasis in our study, the maximum bile duct diameter was evaluated as $8 \mathrm{~mm}$; this was in the patient with cholecystectomy. TAUS has a low sensitivity in detecting CBD stones. However, it is more reliable in detecting dilation in the bile duct due to choledocholithiasis [14,15].

ERCP is the reference diagnostic method in biliary obstruction [16]. In patients with obstructive jaundice, the ERCP failure rate is about 6-7\% [17]. The rate we found in our study was $8.33 \%$. This rate is slightly higher than reported. ERCP is recommended in patients with cholelithiasis and with high risk of choledocholithiasis before the operation. In patients with ERCP failure, EUS and MRCP are recommended [10]. In our opinion, it seems more rational to perform MRCP before ERCP in order to give a broad overview and detect lesions other than choledocholithiasis.

There are some limitations in our study. Nonparametric tests were used for comparison of the groups when the number was limited in the group of patients without choledocholithiasis. There was a delay between MRCP and ERCP because it was a retrospective study. The average delay was 5.46 days and in some of the patients it prolonged to 15 days. During this time interval, although it is a low probability, the persistence of stones in the bile duct might have changed, and this situation might have caused false negative results.

\section{Conclusions}

As a result, in this study we found a high level of interobserver agreement in evaluating MRCP. MRCP has a high sensitivity in detecting choledocholithiasis. However, the specificity found in this study was comparatively low, and this may be due to artefacts caused by high magnetic field. Further long-term studies are needed.

\section{Conflict of interest}

The authors report no conflict of interest. 


\section{References}

1. Makmun D, Fauzi A, Shatri H. Sensitivity and specificity of magnetic resonance cholangiopancreatography versus endoscopic ultrasonography against endoscopic retrograde cholangiopancreatography in diagnosing choledocholithiasis: the Indonesian experience. Clin Endosc 2017; 50: 486-490.

2. Badger WR, Borgert AJ, Kallies KJ, Kothari SN. Utility of MRCP in clinical decision making of suspected choledocholithiasis: An institutional analysis and literature review. Am J Surg 2017; 214: 251-255.

3. Aydelotte JD, Ali J, Huynh PT, et al. Use of magnetic resonance cholangiopancreatography in clinical practice: not as good as we once thought. J Am Coll Surg 2015; 221: 215-219.

4. Polistina FA, Frego M, Bisello M, et al. Accuracy of magnetic resonance cholangiography compared to operative endoscopy in detecting biliary stones, a single center experience and review of literature. World J Radiol 2015; 28: 70-78.

5. Richard F, Boustany M, Britt LD. Accuracy of magnetic resonance cholangiopancreatography for diagnosing stones in the common bile duct in patients with abnormal intraoperative cholangiograms. Am J Surg 2013; 205: 371-373.

6. Demartines N, Eisner L, Schnabel K, et al. Evaluation of magnetic resonance cholangiography in the management of bile duct stones. Arch Surg 2000; 135: 148-152.

7. Merkle EM, Dale BM, Paulson EK. Abdominal MR imaging at 3T. Magn Reson Imaging Clin N Am 2006; 14: 17-26.

8. Nandalur KR, Hussain HK, Weadock WJ, et al. Possible biliary disease: diagnostic performance of high-spatial-resolution isotropic 3D T2-weighted MRCP. Radiology 2008; 249: 883-890.
9. Sudholt $\mathrm{P}$, Zaehringer C, Urigo C, et al. Comparison of optimized 3D-SPACE and 3D-TSE sequences at 1.5T MRCP in the diagnosis of choledocholithiasis. Rofo 2015; 187: 467-471.

10. Maple JT, Ben-Menachem T, Anderson MA, et al. The role of endoscopy in the evaluation of suspected choledocholithiasis. Gastrointest Endosc 2010; 71: 1-9.

11. Parulekar SG. Ultrasound evaluation of bile duct size. Radiology 1979; 133: 703-707.

12. Bachar GN, Cohen M, Belenky A, et al. Effect of aging on the adult extrahepatic bile duct: a sonographic study. J Ultrasound Med 2003; 22: 879-882.

13. Baron RL, Stanley RJ, Lee JKT, et al. A prospective comparison of the evaluation of biliary obstruction using computed tomography and ultrasonography. Radiology 1982; 145: 91-98.

14. Lapis JL, Orlando RC, Mittelstaedt CA, et al. Ultrasonography in the diagnosis of obstructive jaundice. Ann Intern Med 1978; 89: 61-63.

15. Einstein DM, Lapin SA, Ralls PW, et al. The insensitivity of sonography in the detection of choledocholithiasis. Am J Roentgenol 1984; 142: 725-728.

16. Kaltenthaler EC, Walters SJ, Chilcott J, et al. MRCP compared to diagnostic ERCP for diagnosis when biliary obstruction is suspected: a systematic review. BMC Med Imaging 2006; 6: 9.

17. Giovannini M, Bories E, Napoleon B, et al. 855 multicenter randomized phase II study: percutaneous biliary drainage vs EUS guided biliary drainage: results of the intermediate analysis. Gastrointest Endosc 2015; 81: 174. 